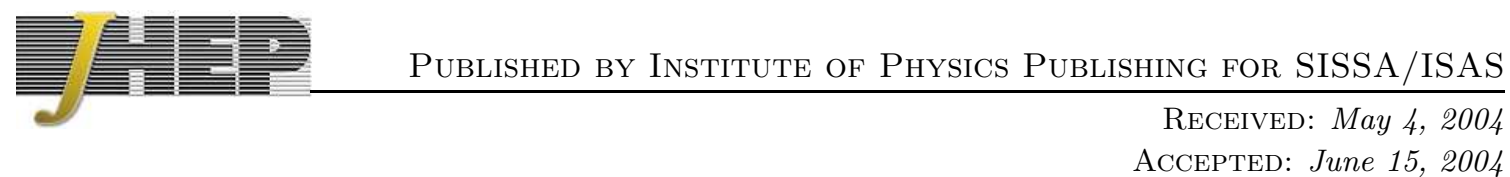

\title{
Notes on $\mathrm{SU}(3)$ structures in type IIB supergravity
}

\author{
Andrew R. Frey \\ California Institute of Technology \\ Mail Stop 452-48, Pasadena, CA 91125, U.S.A. \\ E-mail: frey@theory.caltech.edu
}

\begin{abstract}
We study solutions of type IIB supergravity with an SU(3) structure group and four dimensional Poincaré invariance and present relations among the bosonic fields which follow from the supersymmetry variations. We make explicit some results which also follow from the more general case of an $\mathrm{SU}(2)$ structure and give some short comments applicable to general supersymmetric solutions. We also provide simplified relations appropriate for duals of gauge theory renormalization group flows, and use these to derive the supergravity solution for a bound state of $(p, q) 5$-branes and D3-branes.
\end{abstract}

KeYwords: p-branes, Superstring Vacua, Supergravity Models 


\section{Contents}

1. Introduction 1

2. Constraints from $\mathrm{SU}(3)$ Structure 2

2.1 Normalization

2.2 Complex structure integrability

2.3 Relations among supergravity fields and geometry

2.4 Other concerns 6

3. Radial flow 7

4. $(p, q)$ 5/D3 bound state 8

5. Summary 10

A. Conventions and identities 11

\section{Introduction}

Starting with the analysis of heterotic string compactifications [1], 2, it has been known that the conditions for unbroken supersymmetry, that is, the vanishing of the supersymmetry variations of the supergravity fermions, are often much easier to solve than the equations of motion. Indeed, there are many recent examples in which the supersymmetry variations have been used to find interesting supergravity duals of gauge theories. ${ }^{1}$ Therefore, and also because classically supersymmetric compactifications are important candidates for the vacuum of string theory, a systematic analysis of the supersymmetry conditions is of great intrinsic interest.

Such an analysis has been carried out for M-theory, 11 dimensional supergravity, (and by extension type IIA 10 dimensional supergravity) over the past decade, beginning with [3]-[5]. Very great progress has been made recently by understanding the importance of the structure group in supersymmetry; analysis of M-theory solutions can be found in [6]-17].

The situation in type IIB supergravity has until recently focused much more on specific solutions and particularly simple classes of solutions. Of great importance have been the type B solutions, which have a warped Calabi-Yau geometry, imaginary self-dual and primitive 3 -forms, and a 5 -form determined by the warp factor; the supersymmetry conditions in these solutions were studied in [18]-21]. Compactified type B solutions have been

\footnotetext{
${ }^{1}$ There is a very large literature of examples, and it is beyond the scope of this paper to list them all. We will cite some specific examples as they come up.
} 
studied extensively; see [22] for a review. There is also a class, type A, of solutions dual to the heterotic solutions of [2], which have been analyzed in the group structure language for all supergravities in [23]. (Some compactified type A (and S-dual) solutions in type IIB string theory are discussed in [24, 25]; we do not discuss the widely studied heterotic compactifications here.) Then [26] discussed a more general class of solutions which interpolate between the type B class and the S-dual of the type A solutions, which include D5-brane solutions. Recently, ${ }^{2}$ [27] presented an analysis of $\mathrm{SU}(2)$ structures in type IIB supergravity; this class of solutions includes type A and B solutions as well as particular solutions corresponding to polarized 5-branes [28, 29]. This paper will report the conditions required for $\mathrm{SU}(3)$ structures in IIB supergravity, assuming a four dimensional Poincaré invariance. Although SU(3) structures are a subset of $\mathrm{SU}(2)$ structures, the constraints relating different fields degenerate for a pure $\mathrm{SU}(3)$ structure, so it is useful to have a direct analysis (the precise nature of the degeneration is explained in [26] and will be discussed in section 3 ). (Note that $\mathrm{SU}(3)$ structures in type IIB supergravity have appeared before in studies of mirror symmetry [30].)

We begin in section 2 by giving the supersymmetry variations in string frame and then converting them to relations among the bosonic fields and $\mathrm{SU}(3)$ structure. We present those relations in 2.3 after showing in section 2.2 that the complex structure is integrable, and we make a few general comments in 2.4. In section 3, we integrate the scalar relations in the case that all scalars depend only on one coordinate, such as a radius. These results could be helpful for finding supergravity duals of renormalization group flows. Finally, in section $⿴$, we use our results to derive the supergravity solution for a bound state of a $(p, q)$-brane and D3-branes.

\section{Constraints from SU(3) Structure}

The fermion supersymmetry variations for ten dimensional IIB supergravity are given in [31, 32]. We use the modern string theory conventions, in which the variations are [33, 34]

$$
\begin{aligned}
\delta \lambda= & \frac{1}{2}\left(\partial_{M} \phi-i e^{\phi} \partial_{M} C\right) \Gamma^{M} \varepsilon+\frac{1}{24}\left(i e^{\phi} \tilde{F}-H\right)_{M N P} \Gamma^{M N P} \varepsilon^{*} \\
\delta \psi_{M}= & \left(\nabla_{M}+\frac{i}{8} e^{\phi} \partial_{N} C \Gamma^{N} \Gamma_{M}+\frac{i}{16 \cdot 5 !} e^{\phi} \tilde{F}_{N P Q R S} \Gamma^{N P Q R S} \Gamma_{M}\right) \varepsilon- \\
& -\left(\frac{1}{8} H_{M N P} \Gamma^{N P}+\frac{i}{48} e^{\phi} \tilde{F}_{N P Q} \Gamma^{N P Q} \Gamma_{M}\right) \varepsilon^{*} .
\end{aligned}
$$

The supersymmetry parameter $\varepsilon$ is a 10D Weyl spinor of positive chirality; the rest of the conventions are explained in A.

Since we are working in a vacuum with $4 \mathrm{D}$ Poincaré invariance, the most general ansatz we can take is

$$
\begin{aligned}
d s^{2} & =e^{2 A} \eta_{\mu \nu} d x^{\mu} d x^{\nu}+g_{m n} d x^{m} d x^{n} \\
\tilde{F}_{\mu \nu \lambda \rho m} & =e^{-4 A} \epsilon_{\mu \nu \lambda \rho} \partial_{m} h .
\end{aligned}
$$

\footnotetext{
${ }^{2}$ In fact, this paper was in preparation when the results of 27 appeared.
} 
All other form components and all field dependences are in the internal directions. We include the factor $e^{-4 A}$ in the 5 -form so that $\tilde{F}_{\mu \nu \lambda \rho m}$ is an external derivative. Field strengths without tildes are just external derivatives, while tildes denote the addition of Chern-Simons terms. The only definition of importance to us is $\tilde{F}_{3}=F_{3}-\mathrm{CH}_{3}$, as the 5 -form is given through its self-duality.

\subsection{Normalization}

In this subsection and the following one, we will emphasize two points which were not stated explicitly in [27]. The first point regards the normalization of the spinors. If we start by taking

$$
\varepsilon=e^{A / 2}\left(\zeta \otimes \chi_{+}+\zeta^{*} \otimes \chi_{-}\right),
$$

where $\zeta$ is a $4 \mathrm{D}$ Weyl spinor of positive chirality and $\chi_{ \pm}$are $6 \mathrm{D}$ Weyl spinors of respective chirality, the internal and external gravitino variations (2.2) combine to give

$$
\begin{aligned}
& {\left[\nabla_{m}+\frac{i}{4} e^{\phi} \partial_{m} C-\frac{1}{2} \partial_{n} A \gamma_{m}{ }^{n}+\frac{1}{4} e^{\phi-4 A} \partial_{n} h \gamma_{m}^{n}\right] \chi_{+}-\frac{1}{8}\left(H+i e^{\phi} \tilde{F}\right)_{m n p} \gamma^{n p} \chi_{-}^{*}=0} \\
& {\left[\nabla_{m}+\frac{i}{4} e^{\phi} \partial_{m} C-\frac{1}{2} \partial_{n} A \gamma_{m}^{n}-\frac{1}{4} e^{\phi-4 A} \partial_{n} h \gamma_{m}^{n}\right] \chi_{-}-\frac{1}{8}\left(H+i e^{\phi} \tilde{F}\right)_{m n p} \gamma^{n p} \chi_{+}^{*}=0 .}
\end{aligned}
$$

We can immediately see that

$$
\begin{aligned}
\nabla_{m}\left(\chi_{+}^{\dagger} \chi_{+}+\chi_{-}^{\dagger} \chi_{-}\right)= & \frac{1}{8}\left(H+i e^{\phi} \tilde{F}\right)_{m n p}\left(\chi_{+}^{\dagger} \gamma^{n p} \chi_{-}^{*}+\chi_{-}^{\dagger} \gamma^{n p} \chi_{+}^{*}\right)- \\
& -\frac{1}{8}\left(H-i e^{\phi} \tilde{F}\right)_{m n p}\left(\chi_{-}^{T} \gamma^{n p} \chi_{+}+\chi_{+}^{T} \gamma^{n p} \chi_{-}\right) .
\end{aligned}
$$

This vanishes by the symmetry properties of the $\gamma^{m}$ matrices. We easily see, therefore, that the $\mathrm{SU}(2)$ structure spinors of [27] (in the notation of that paper) satisfy

$$
e^{-A}\left(|a|^{2}+|b|^{2}+|c|^{2}\right)=\text { constant } \equiv 1 .
$$

(The warp factor must be included because [27 does not scale the spinor by an overal power of the warp factor.)

In an $\mathrm{SU}(3)$ structure, there is only one linearly independent $6 \mathrm{D}$ spinor, which corresponds to $c \rightarrow 0$ in the $\mathrm{SU}(2)$ structure notation of [27]. We can therefore write our supersymmetry parameters as

$$
\varepsilon=e^{A / 2}\left(\cos (\alpha / 2) e^{i \beta / 2} \zeta \otimes \chi+\sin (\alpha / 2) e^{i \beta / 2} \zeta^{*} \otimes \chi^{*}\right)
$$

with $\chi$ a positive chirality $6 \mathrm{D}$ spinor with $\chi^{\dagger} \chi=1$.

A number of recent papers [35, 28, 36, 29] have used an "algebraic spinor" approach to derive supergravity duals to renormalization group flows. In this approach, the spinors are specified by projector equations; the projector corresponding to (2.8) (that is, such that $P \varepsilon=\varepsilon)$ is

$$
P=\frac{1}{2}\left[1+\cos \alpha \gamma_{(\hat{6})}+\sin \alpha e^{i \beta} *\right]
$$


where $\gamma_{(\hat{6})}$ is the six dimensional chirality (alternately, the four dimensional chirality can be used) and $*$ is the complex conjugation operator. Additional projectors are necessary to reduce the number of supersymmetries to $\mathcal{N}=1$; these simply align the spins of $\chi$ (see the final paragraph of 2.2. .

\subsection{Complex structure integrability}

The other point which we wish to make explicit concerns the integrability of the almost complex structure (ACS). Any SU(3) structure has an almost complex structure tensor as well as a $(3,0)$ form defined with respect to that ACS; these forms give an alternate definition of the $\mathrm{SU}(3)$ structure in $6 \mathrm{D}$. Defining the ACS

$$
J_{m}^{n}=-i \chi^{\dagger} \gamma_{m}^{n} \chi
$$

Fierz identities (see appendix A show that it indeed squares to -1 . See, for example, [23 for a nice review of different group structures in different dimensions.

The ACS is integrable if the Nijenhuis tensor $N_{m n}{ }^{p}=J_{m}^{q} \nabla_{[q} J_{n]}^{p}-J_{n}^{q} \nabla_{[q} J_{m]}{ }^{p}$ vanishes. From (2.5), we find

$$
\begin{aligned}
\nabla_{m} J_{n}{ }^{p}= & -\left(\partial_{q} A-\frac{1}{2} \cos \alpha e^{\phi-4 A} \partial_{q} h\right)\left(g_{m n} J^{p q}-\delta_{m}^{p} J_{n}{ }^{q}+\delta_{n}^{q} J_{m}{ }^{p}-g^{p q} J_{m n}\right)+ \\
& +\frac{1}{2} T_{m n}{ }^{r} J_{r}{ }^{p}-\frac{1}{2} T_{m r}^{p} J_{n}{ }^{r} \\
T_{m n p} \equiv & \sin \alpha\left(\cos \beta H+\sin \beta e^{\phi} \tilde{F}\right)_{m n p} .
\end{aligned}
$$

The flux tensor $T$ acts like a torsion in the connection, and the other terms in $\nabla J$ are similar to those generated in a connection by rescaling the metric. In fact, it is a short calculation to see that the terms with $A$ and $h$ contribute nothing to the Nijenhuis tensor; the only contribution is from the torsion.

However, one linear combination of equations that result from the dilatino variation (2.1) is

$$
\begin{aligned}
& \frac{1}{12} T_{m n p} \gamma^{m n p} \chi= \\
& \quad=\left[(\cos (2 \beta)+i \cos \alpha \sin (2 \beta)) \partial_{m} \phi+(\sin (2 \beta)-i \cos \alpha \cos (2 \beta)) e^{\phi} \partial_{m} C\right] \gamma^{m} \chi .
\end{aligned}
$$

Once we have equation (2.13), [2] shows that the torsion contributes nothing to the Nijenhuis tensor. Therefore, for an SU(3) structure, the complex structure is always integrable! In fact, this follows from equation (3.33) in [27]; this is a more explicit statement and proof. (This proof also ties up a loose end in [26].)

With an integrable complex structure, there is a holomorphic atlas, so we can write the defining relation for a Hermitean metric $J_{i \bar{\jmath}}=i g_{i \bar{\jmath}}$ in the complex coordinates. This, along with 2.10), implies that $\gamma_{i} \chi=\gamma^{\bar{\imath}} \chi=0$. 


\subsection{Relations among supergravity fields and geometry}

The vanishing of the fermion variations (2.1), (2.2) implies a number of constraints on the supergravity fields and the $\mathrm{SU}(3)$ structure of the manifold. Here, we briefly report those constraints, which are a special case of those reported in [27 for SU(2) structures. We write these constraints directly in terms of the supergravity fields, rather than decomposing the fields according to the $\mathrm{SU}(3)$ structure.

There are four independent equations relating the scalars $\alpha, \beta, A, h, \phi$, and $C$. These relations, in the complex coordinates defined by $J$, are

$$
\begin{aligned}
b_{1} \partial A+\frac{1}{4} a_{1}\left(i e^{\phi} \partial C-e^{\phi-4 A} \partial h\right) & =0 \\
a_{1} \partial A-\frac{1}{2} a_{1} \partial \phi-\frac{1}{4} b_{1}\left(i e^{\phi} \partial C+e^{\phi-4 A} \partial h\right) & =0 \\
\frac{1}{2} \sin (2 \alpha) \partial \alpha-\cos \alpha\left(a_{2} \partial \phi+i b_{2} e^{\phi} \partial C\right) & =0 \\
\sin ^{2} \alpha\left(\partial \beta+\frac{1}{2} e^{\phi} \partial C+\frac{i}{2} e^{\phi-4 A} \partial h\right)+\cos \alpha\left(i b_{2} \partial \phi-a_{2} e^{\phi} \partial C\right) & =0
\end{aligned}
$$

where

$$
\begin{array}{ll}
a_{1}=\cos \alpha \cos \beta-i \sin \beta, & b_{1}=\cos \beta-i \cos \alpha \sin \beta \\
a_{2}=\cos \alpha \cos (2 \beta)-i \sin (2 \beta), & b_{2}=\cos (2 \beta)-i \cos \alpha \sin (2 \beta) .
\end{array}
$$

In the third equation of (2.13), we have left in the overall factor of $\cos \alpha$ to demonstrate how the relations can degenerate at loci in the space of spinors. Finally, we note that it is simple to translate these equations to real coordinates by using the complex structure. We simply take the holomorphic derivative to the exterior derivative $\partial f \rightarrow d f$ when no factors of $i$ are present and similarly $i \partial f \rightarrow J_{m}{ }^{n} \partial_{n} f d x^{m}$. The integrability conditions for (2.13) are quite complicated, and we have not done a full analysis. However, in certain cases, such as $\alpha \rightarrow 0, \pi / 2$ or $\beta \rightarrow 0, \pi / 2$, the integrability conditions (and scalar relations) simplify considerably. The only new condition introduced in any of those cases is that $\partial \phi \wedge \partial C=0$. In section 3 , we will discuss a class of solutions in which we can integrate (2.13) explicitly.

There are numerous algebraic and differential relations for the 3-forms and $\mathrm{SU}(3)$ structure. We start by defining the $(3,0)$ form of the structure,

$$
\Omega_{m n p}=\chi^{T} \gamma_{m n p} \chi
$$

Then it is straightforward to see

$$
H_{m n p} \Omega^{m n p}=\tilde{F}_{m n p} \Omega^{m n p}=0
$$

and the conjugate equations, so there are no $(3,0)$ or $(0,3)$ components of the fluxes. There are also the following relations for the fluxes:

$$
\begin{aligned}
& \left(\cos \alpha \cos \beta \delta_{m}^{n}-\sin \beta J_{m}{ }^{n}\right) \partial_{n} \phi+\left(\cos \alpha \sin \beta \delta_{m}^{n}+\cos \beta J_{m}{ }^{n}\right) e^{\phi} \partial_{n} C=\frac{1}{4} \sin \alpha e^{\phi} \tilde{F}_{m n p} J^{n p} \\
& \left(\cos \alpha \sin \beta \delta_{m}^{n}+\cos \beta J_{m}{ }^{n}\right) \partial_{n} \phi-\left(\cos \alpha \cos \beta \delta_{m}^{n}-\sin \beta J_{m}{ }^{n}\right) e^{\phi} \partial_{n} C=\frac{1}{4} \sin \alpha H_{m n p} J^{n p}
\end{aligned}
$$


and

$$
\begin{aligned}
-\frac{1}{4}\left(\sin \beta H-\cos \beta e^{\phi} \tilde{F}\right)_{m n p} J^{n p} & =\partial_{m} \alpha \\
-\frac{1}{4} \cos \alpha\left(\cos \beta H+\sin \beta e^{\phi} \tilde{F}\right)_{m n p} J^{n p} & =\sin \alpha\left(\partial_{m} \beta+\frac{1}{2} e^{\phi} \partial_{m} C+\frac{1}{2} e^{\phi-4 A} \partial_{n} h J_{m}{ }^{n}\right) .
\end{aligned}
$$

After a bit more work and application of equation (A.7),

$$
\begin{gathered}
0=\left[2 \sin \alpha e^{\phi-4 A} g_{m[n} \partial_{p]} h+\cos \alpha\left(\cos \beta H+\sin \beta e^{\phi} \tilde{F}\right)_{m n p}+\right. \\
\left.+i\left(\sin \beta H-\cos \beta e^{\phi} \tilde{F}\right)_{m n p}\right]\left(\delta_{q}^{n}+i J_{q}{ }^{n}\right)\left(\delta_{r}^{p}+i J_{r}{ }^{p}\right) .
\end{gathered}
$$

In the type $\mathrm{B}, \alpha=0$, ansatz, this equation requires that $G=\tilde{F}-i e^{-\phi} H$ is $(1,2)$ only. This is opposite the usual convention for choice of complex coordinates.

The other relations are differential in the complex structure and $(3,0)$ form (here $T$ is the torsion part of the 3 -forms, as defined in equation (2.12)):

$$
\begin{aligned}
d J= & \left(2 d A-\cos \alpha e^{\phi-4 A} d h+2 \cos (2 \beta) d \phi+2 \sin (2 \beta) e^{\phi} d C\right) \wedge J+ \\
& +\star\left[T-2 \cos \alpha\left(\sin (2 \beta) d \phi-\cos (2 \beta) e^{\phi} d C\right) \wedge J\right] \\
\nabla_{n} J_{m}{ }^{n}= & \left(4 \partial_{n} A-2 \cos \alpha e^{\phi-4 A} \partial_{n} h+2 \cos (2 \beta) \partial_{n} \phi+2 \sin (2 \beta) e^{\phi} \partial_{n} C\right) J_{m}{ }^{n}+ \\
& +2 \cos \alpha\left(\sin (2 \beta) \partial_{m} \phi-\cos (2 \beta) e^{\phi} \partial_{m} C\right) \\
d \Omega= & {\left[3 d A-\frac{3}{2} \cos \alpha e^{\phi-4 A} d h+i \cos \alpha\left(d \beta+\frac{1}{2} e^{\phi} d C\right)+\right.} \\
& \left.+2 \cos (2 \beta) d \phi+2 \sin (2 \beta) e^{\phi} d C\right] \wedge \Omega .
\end{aligned}
$$

There is no separate equation for $d \star_{6} \Omega$ because $\Omega$ satisfies a self-duality relation in the internal space. Note that it is often possible to integrate the $d h$ terms, in which case the $d A, d h$ terms both can be interpreted as due to rescaling the six dimensional metric.

\subsection{Other concerns}

The relations described in section 2.3, while containing information about the supersymmetry of a particular string vacuum, do not suffice to specify the solution entirely. It is also necessary to apply the Bianchi identities for the field strengths and to check that the solution satisfies the equations of motion. The necessary Bianchi identities are

$$
\begin{aligned}
d H & =0, \quad d F_{3}=0 \quad \text { or } \quad d \tilde{F}_{3}=-d C \wedge H \\
d \tilde{F}_{5} & =H_{3} \wedge F_{3} .
\end{aligned}
$$

Note that the 5 -form Bianchi identity is also an equation of motion due to the self-duality of the 5 -form. 
The reason we should also check the equations of motion is that not all the equations of motion are necessarily contained in the commutators of the supersymmetry variations. This issue was discussed in [6] for the case of M-theory. However, in IIB supergravity, [32] was able to derive all the equations of motion by using the supersymmetry algebra. It seems, therefore, that because the 5-form Bianchi is also an equation of motion, supersymmetry of a type IIB background, along with the Bianchi identities, may imply that it solves the equations of motion.

\section{Radial flow}

In this section, we integrate the scalar relations (2.13) in a case that should be relevant for renormalization group flows in string-gauge dualities as well as for supersymmetric brane bound states. Specifically, we consider the case that all the scalars depend only on a single (real) dimension, such as a radial direction. This ansatz could be useful for finding supergravity solutions relating the supergravity backgrounds of [37] and [38], for example, as both are $\mathcal{N}=1$ backgrounds with $\mathrm{SU}(3)$ structure (see [39] and [19, 20] for supersymmetry analysis, respectively).

Once we specify that the scalars depend only on a single direction $r$, we can separate (2.13) into real and imaginary parts by factoring out $\partial r / \partial z^{i}$. The eight equations we have are more than enough to define all the scalars in terms of one of the angles $\alpha, \beta$, and in fact the extra equations are compatible with the following solutions. In determining the proportionality factors in the following, we assumed that as $r \rightarrow \infty, \beta \rightarrow \theta$ and $\alpha \rightarrow \pi / 2-\theta^{\prime}$ with $\theta, \theta^{\prime} \neq 0, \pi / 2$. We also took $\phi \rightarrow \ln g_{s}, C \rightarrow 0$, and $A \rightarrow 0$ as boundary conditions; these are appropriate for the asymptotically flat region around a brane bound state. However, it is possible to adjust the proportionality constants to allow for other boundary conditions, as would be the case in string-gauge dualities. We have

$$
\begin{aligned}
\cos \alpha & =\sin \theta^{\prime} \cot \theta \tan \beta \\
e^{\phi} & =g_{s} \frac{\sin (2 \theta)}{\sin (2 \beta)} \\
C & =\frac{\tan \theta}{g_{s}}\left(1-\frac{\sin ^{2} \beta}{\sin ^{2} \theta}\right) \\
e^{2 A} & =\frac{\sin \theta \cot \theta^{\prime}}{\sin \beta \tan \alpha}=\frac{\cos \theta^{\prime} \cos \theta}{\sin \alpha \cos \beta} \\
h & =\frac{1}{g_{s}} \cot ^{2} \theta^{\prime} \sin \theta^{\prime} \cot ^{2} \alpha+h_{0}
\end{aligned}
$$

with $h_{0}$ some constant (which we can fix to zero by a gauge transformation).

This is an appropriate point to emphasize how the scalar relations (2.13) degenerate in certain limits, which makes the integrated scalars (3.1) singular. The "renormalization" necessary to interpret (3.1) in such a limit is discussed in detail in [26, where it was argued that the proportionality constants (i.e., the angles $\theta, \theta^{\prime}$ ) can absorb zeros and infinities. As an example, suppose that $\beta(x)=\pi / 2-\epsilon z(x)$, where $\epsilon \rightarrow 0$ in the limit we consider. To 
renormalize (3.1), then let also $\theta=\pi / 2-\epsilon$. By being careful, we get the new relations

$$
\begin{aligned}
\cos \alpha & =\sin \theta^{\prime} z^{-1} \\
e^{\phi} & =g_{s} z^{-1}=\frac{g_{s}}{\sin \theta^{\prime}} \cos \alpha, \quad C=0 \\
e^{2 A} & =\frac{\cot \theta^{\prime}}{\tan \alpha},
\end{aligned}
$$

and $h$ is the same as in (3.1). These results are in fact identical to those of [26, which directly considered supersymmetry parameters with varying $\alpha$ and $\beta=\pi / 2$. Note, however, that here we have seen that vanishing asymptotic $C$ requires $C$ to vanish everywhere, which seems to restrict the varying $C$ solutions of [26].

We see, therefore, how limits which turn off some variation of the supersymmetry parameter are singular. In fact, this should be precisely true for the restriction of an $\mathrm{SU}(2)$ structure to an $\mathrm{SU}(3)$ structure. This is one reason that it is useful to have a direct analysis of the $\mathrm{SU}(3)$ structure case.

\section{4. $(p, q) 5 / \mathrm{D} 3$ bound state}

Now we will use the integrated scalars (3.1) above to derive the supergravity solution corresponding to a bound state of $(p, q) 5$-branes and D3-branes. This solution has been obtained previously by a clever use of zero modes in the equations of motion [40] and by application of dualities to known brane solutions 41, 42. The equivalence of these solutions was demonstated in 433. After we have determined the solution explicitly, we can compare to the asymptotically flat bound state solution of [41, 42].

We start by specifying the six dimensional metric. These six dimensions separate into those parallel and transverse to the $(p, q) 5$-brane. Since we are working with the flat space solutions, the orthogonal directions should have an $\mathrm{SO}(4)$ symmetry for a singlecenter solution. Similarly, the two directions parallel to the 5-brane but orthogonal to the D3-branes (i.e., the directions of the 5-brane worldvolume field strength) should be translationally invariant. Restricting $a, b=4,5$ along the 5 -brane and $m, n=6-9$ orthogonal, the metric becomes

$$
d s_{6}^{2}=e^{2 B_{1}} \tilde{g}_{a b} d x^{a} d x^{b}+e^{2 B_{2}} \delta_{m n} d x^{m} d x^{n} .
$$

Then all of the supergravity scalars, $\alpha, \beta$, and the warp factors $A, B_{1,2}$ depend only on the radial direction $r^{2}=\delta_{m n} x^{m} x^{n}$. The complex structure can be calculated by wedging the vielbein $J=e_{\hat{4}} \wedge e_{\hat{5}}+e_{\hat{6}} \wedge e_{\hat{7}}+e_{\hat{8}} \wedge e_{\hat{9}}$. (Hats denote tangent space indices.)

Because the 3 -forms $H, F$ have vanishing Bianchis (up to sources at the origin), their integral over $S^{3}$ s concentric with the origin is independent of radius; we normalize as

$$
\oint_{S^{3}} H=2 p \Omega_{S^{3}}, \quad \oint_{S^{3}} F=2 q \Omega_{S^{3}}
$$


where $\Omega_{S^{3}}$ is the volume of a unit $S^{3}{ }^{3}$ The allowed components are therefore

$$
\begin{aligned}
H_{m n p} & =e^{-2 B_{2}} p \epsilon_{m n p}{ }^{q} \partial_{q} g, & F_{m n p} & =e^{-2 B_{2}} q \epsilon_{m n p}{ }^{q} \partial_{q} g, \quad g=\frac{1}{r^{2}} \\
H_{r 45} & =e^{-2 B_{1}} \epsilon_{45} \partial_{r} g_{1}, & F_{r 45} & =e^{-2 B_{1}} \epsilon_{45} \partial_{r} g_{2} .
\end{aligned}
$$

Consider (2.20). The $m=r$ component along with the scalar relations (3.1) immediately give (dropping the integration constants by gauge choice)

$$
g_{1}=-g_{s} \tan \theta g_{2}
$$

Then the same component of $(2.19)$ and (3.1) can be integrated to give

$$
g_{1}=\frac{\sin \theta}{\sin \left(2 \theta^{\prime}\right)} \cos (2 \alpha), \quad g_{2}=-\frac{\cos \theta}{g_{s} \sin \left(2 \theta^{\prime}\right)} \cos (2 \alpha) \text {. }
$$

We can continue by considering (2.22). The component along the three angular directions vanishes on both sides, $d J$ by computation, and the right hand side by (3.1) and (4.4). The component along the radius and two of the angles yields a differential equation for the $B_{2}$ warp factor; assuming asymptotic flatness gives

$$
e^{2 B_{2}}=\frac{\sin ^{2} \theta}{\cos \theta^{\prime} \cos \theta} \frac{\sin \alpha \cos \beta}{\sin ^{2} \beta} .
$$

The $r 45$ component gives a differential equation for $B_{1}$; however, this equation exhibits the radius explicitly due to the appearance of the fluxes. To solve it, we need to know the radial dependence of the angle $\beta$ (and therefore all the other scalars).

This we find from the angular components of (2.19), (2.20). Because $\alpha$ depends only on $r$, we find immediately from (2.19) that $\tan \theta=q g_{s} / p$. Then we can integrate (2.20) to find

$$
\sin \beta=\frac{r \sin \theta}{\left[r^{2}+p \cos \theta \cos \theta^{\prime}\right]^{1 / 2}} .
$$

We can now integrate the equation for $B_{1}$; coincidentally, the explicit $r$ dependence now can be reabsorbed into $\beta$, so

$$
e^{2 B_{1}}=\frac{\cos \theta}{\cos \theta^{\prime}} \frac{\sin \alpha}{\cos \beta} .
$$

We can now write the radial dependence of all the scalars, warp factors, and fluxes.

The final thing to determine is the asymptotic angle $\theta^{\prime}$. As discussed in section 2.4, we should also have to apply the 5 -form Bianchi identity to solve the equations of motion. As a substitute, however, we will note that we want a brane bound state with $n$ units of D3-brane charge (using the same normalization as in equation (4.2)). Therefore, asymptotically, where $H \wedge F$ vanishes, we should have

$$
\oint_{T^{2} \times S^{3}} \tilde{F}_{5}=2 n \Omega_{S^{3}} V_{T^{2}}
$$

\footnotetext{
${ }^{3}$ This normalization absorbs the 5 -brane charge into $p, q$.
} 
where $V_{T^{2}}$ is the volume of the torus wrapped by the 5-branes. ${ }^{4}$ Using the self-duality of the 5 -form, this condition can be written as

$$
e^{2 B_{1}} e^{2 B_{2}} e^{-4 A} \partial_{r} h=\frac{2 n}{r^{3}},
$$

Comparing to the radial behavior of the scalars (4.7) then yields $\tan \theta^{\prime}=n g_{s} / \sqrt{p^{2}+q^{2} g_{s}^{2}}$.

We can also check that the remaining constraints are satisfied. Indeed, (2.17), (2.18) are just linear combinations of (2.19), (2.20) if we use (3.1). We will not explicitly discuss (2.21), (2.23), (2.24), but they are straightforward, though slightly tedious, to check.

Finally, we note that we can compare our solution to that obtained by dualities in 41 , 42] (with vanishing asymptotic RR scalar). That solution is written in terms of three warp factors, $H, H^{\prime}, H^{\prime \prime}$ in the notation of [41, 42. By comparing the radial dependence, it is easy to see that

$$
H=\tan \theta^{\prime} \tan \alpha, H^{\prime}=\tan ^{2} \theta \cot ^{2} \beta=\frac{\sin ^{2} \theta^{\prime}}{\cos ^{2} \alpha}, \quad H^{\prime \prime}=\frac{\sin ^{2} \theta}{\sin ^{2} \beta} .
$$

Then the solutions match exactly (up to sign conventions). While this bound state solution has been obtained before, both from dualities and the equations of motion, it is worth noting that our methods may be more easily generalized to geometries with less symmetry and also to boundary conditions more appropriate for gauge-string theory dualities.

\section{Summary}

In this paper, we have presented constraints on solutions of type IIB supergravity with four dimensional Poincaré invariance and $\mathcal{N}=1$ supersymmetry based on an $\mathrm{SU}(3)$ structure. The $\mathrm{SU}(3)$ structure is a limit of $\mathrm{SU}(2)$ structures which have been recently presented in [27]; however, the limit is degenerate, so a direct analysis is desirable. Additionally, $\mathrm{SU}(3)$ structures have nongeneric behavior among SU(2) structures; for example, all SU(3) structures have an integrable complex structure.

In section 3, we presented a solution to the relations among the scalars in the case that all the scalars depend only on a single (radial) direction. It is our hope that this solution, with appropriately modified boundary conditions, will be useful in constructing supergravity duals to renormalization group flows. We have used these results to derive the supergravity solution for the $(p, q) 5 / \mathrm{D} 3$-brane bound state.

\section{Acknowledgments}

I would like to thank M. Caldarelli, G. Dall'Agata, J. Gauntlett, J. Gomis, M. Graña, and M. Schulz for useful communications. This work was supported by the John A. McCone Fellowship in Theoretical Physics at the California Institute of Technology.

\footnotetext{
${ }^{4}$ This seems to be an odd quantization condition; however, we can derive it by considering the eight dimensional theory reduced along the torus. It also happens to give $n$ the same dimensionality as $p, q$.
} 


\section{A. Conventions and identities}

In this paper, we work in string frame with usual string conventions for the field strengths (see [34]). Note that the gravitino, dilatino, and supersymmetry parameter are redefined in transforming from the Einstein frame. Indices $M, N$ are for the full 10 dimensions, $\mu, \nu$ for the 4 Poincaré invariant dimensions, and $m, n$ for the internal dimensions. Hats denote tangent space indices. We work in a signature in which timelike norms are negative.

Our differential form conventions are as follows:

$$
\begin{aligned}
\epsilon_{012 \cdots(d-1)} & =+\sqrt{-g} \text { for } d \text { dimensions } \\
T_{\left[M_{1} \cdots M_{p}\right]} & =\frac{1}{p !}\left(T_{M_{1} \cdots M_{p}} \pm \text { permutations }\right) \\
(\star T)_{M_{1} \cdots M_{d-p}} & =\frac{1}{p !} \epsilon_{M_{1} \cdots M_{d-p}}{ }^{N_{1} \cdots N_{p}} T_{N_{1} \cdots N_{p}} \\
T & =\frac{1}{p !} T_{M_{1} \cdots M_{p}} d x^{M_{1}} \cdots d x^{M_{p}} .
\end{aligned}
$$

Wedges and exterior derivatives are defined consistently with those conventions. We choose $\star \tilde{F}_{5}=\tilde{F}_{5}$ for the self-duality of the 5 -form.

Gamma matrices in tangent space have the algebra $\left\{\Gamma^{\hat{M}}, \Gamma^{\hat{N}}\right\}=2 \eta^{\hat{M} \hat{N}}$. With these conventions, a Majorana basis is real and symmetric for spacelike indices and antisymmetric for time. Gammas can be converted to coordinate indices with the vielbein. We define $\Gamma^{M_{1} \cdots M_{p}}=\Gamma^{\left[M_{1}\right.} \cdots \Gamma^{\left.M_{p}\right]}$. The chirality is given by

$$
\Gamma_{(\widehat{10})}=\Gamma^{\hat{0}} \cdots \Gamma^{\hat{9}}=\frac{1}{10 !} \epsilon_{M_{1} \cdots M_{10}} \Gamma^{M_{1} \cdots M_{10}} .
$$

The gravitino and supersymmetry parameter have positive chirality; this choice agrees with the 5-form self-duality above.

We can decompose the $\Gamma$ matrices as

$$
\Gamma^{\mu}=\gamma^{\mu} \otimes 1, \quad \Gamma^{m}=\gamma_{(\hat{4})} \otimes \gamma^{m}
$$

with 4 and 6 dimensional chirality $\gamma_{(\hat{4})}=-i \gamma^{\hat{0}} \cdots \gamma^{\hat{3}}, \gamma_{(\hat{6})}=i \gamma^{\hat{4}} \cdots \gamma^{\hat{9}}$. The $\gamma^{\mu}$ have the same symmetry and reality properties as $\Gamma^{M}$, while the $\gamma^{m}$ are imaginary and antisymmetric.

A number of $\gamma$ identities are very useful. A comprehensive list of (anti)commutators appears in [44], although there is at least one typographical error. It is necessary to replace

$$
\left[\gamma_{m n p}, \gamma^{r s t}\right]=2 \gamma_{m n p}{ }^{r s t}-36 \delta_{[m n}^{[r s} \gamma_{p]}^{t]}
$$

Additionally,

$$
\gamma_{m n p q}=\frac{i}{2} \gamma_{(\hat{6})} \gamma^{r s} \epsilon_{m n p q r s}, \quad \chi^{\dagger} \gamma_{m n p q r s} \chi=-i \epsilon_{m n p q r s}
$$

for positive chirality $\chi$.

The Fierz identities that we use come from expanding in terms of the complete set of $\gamma$ matrices. Specifically, we find

$$
\chi \chi^{\dagger}=\frac{1}{8}-\frac{i}{16} J_{m n} \gamma^{m n}-\frac{i}{16} J_{m n} \gamma^{m n} \gamma_{(\hat{6})}+\frac{1}{8} \gamma_{(\hat{6})}
$$


for the normalized positive chirality spinor $\chi$ used in the text. This identity can be used to show that $J_{m}{ }^{n} J_{n}{ }^{p}=-\delta_{m}^{p}$ and also that

$$
6 J_{[m n} J_{p q]}=\epsilon_{m n p q}{ }^{r s} J_{r s} .
$$

\section{References}

[1] P. Candelas, G.T. Horowitz, A. Strominger and E. Witten, Vacuum configurations for superstrings, Nucl. Phys. B 258 (1985) 46.

[2] A. Strominger, Superstrings with torsion, Nucl. Phys. B 274 (1986) 253.

[3] K. Becker and M. Becker, M-theory on eight-manifolds, Nucl. Phys. B 477 (1996) 155 hep-th/9605053.

[4] K. Becker, A note on compactifications on $\mathrm{Spin}_{7}$-holonomy manifolds, J. High Energy Phys. 05 (2001) 003 hep-th/0011114.

[5] K. Becker and M. Becker, Compactifying M-theory to four dimensions, J. High Energy Phys. 11 (2000) 029 hep-th/0010282.

[6] J.P. Gauntlett and S. Pakis, The geometry of D $=11$ Killing spinors,. J. High Energy Phys. 04 (2003) 039 hep-th/0212008.

[7] P. Kaste, R. Minasian, M. Petrini and A. Tomasiello, Nontrivial RR two-form field strength and SU(3)-structure, Fortschr. Phys. 51 (2003) 764 hep-th/0301063.

[8] K. Behrndt and C. Jeschek, Fluxes in M-theory on 7-manifolds and G structures, J. High Energy Phys. 04 (2003) 002 hep-th/0302047.

[9] P. Kaste, R. Minasian and A. Tomasiello, Supersymmetric M-theory compactifications with fluxes on seven-manifolds and g-structures, J. High Energy Phys. 07 (2003) 004 hep-th/0303127.

[10] D. Martelli and J. Sparks, G-structures, fluxes and calibrations in M-theory, Phys. Rev. D 68 (2003) 085014 hep-th/0306225.

[11] K. Behrndt and C. Jeschek, Fluxes in M-theory on 7-manifolds: G-structures and superpotential, hep-th/0311119.

[12] G. Dall'Agata and N. Prezas, $N=1$ geometries for $M$-theory and type-IIA strings with fluxes, Phys. Rev. D 69 (2004) 066004 hep-th/0311146.

[13] J.P. Gauntlett, J.B. Gutowski and S. Pakis, The geometry of D $=11$ null Killing spinors, O. High Energy Phys. 12 (2003) 049 hep-th/0311112.

[14] K. Behrndt and M. Cvetič, Supersymmetric intersecting d6-branes and fluxes in massive type-IIA string theory, Nucl. Phys. B 676 (2004) 149 hep-th/0308045.

[15] J.P. Gauntlett, D. Martelli, J. Sparks and D. Waldram, Supersymmetric AdS $S_{5}$ solutions of M-theory, hep-th/0402153.

[16] M. Becker, K. Dasgupta, A. Knauf and R. Tatar, Geometric transitions, flops and non-Kähler manifolds, I, hep-th/0403288.

[17] K. Behrndt and M. Cvetič, General $N=1$ supersymmetric flux vacua of (massive) type-IIA string theory, hep-th/0403049. 
[18] A. Kehagias, New type-IIB vacua and their F-theory interpretation, Phys. Lett. B 435 (1998) 337 hep-th/9805131.

[19] M. Graña and J. Polchinski, Supersymmetric three-form flux perturbations on AdS 5 , Phys. Rev. D 63 (2001) 026001 hep-th/0009211.

[20] S.S. Gubser, Supersymmetry and F-theory realization of the deformed conifold with three-form flux, hep-th/0010010.

[21] M. Graña and J. Polchinski, Gauge/gravity duals with holomorphic dilaton, Phys. Rev. D 65 (2002) 126005 hep-th/0106014.

[22] A.R. Frey, Warped strings: self-dual flux and contemporary compactifications, hep-th/0308156.

[23] J.P. Gauntlett, D. Martelli and D. Waldram, Superstrings with intrinsic torsion, Phys. Rev. D 69 (2004) 086002 hep-th/0302158.

[24] K. Becker and K. Dasgupta, Heterotic strings with torsion, J. High Energy Phys. 11 (2002) 006 hep-th/0209077.

[25] S. Kachru, M.B. Schulz, P.K. Tripathy and S.P. Trivedi, New supersymmetric string compactifications, J. High Energy Phys. 03 (2003) 061 hep-th/0211182.

[26] A.R. Frey and M. Graña, Type IIB solutions with interpolating supersymmetries, Phys. Rev. D 68 (2003) 106002 hep-th/0307142.

[27] G. Dall'Agata, On supersymmetric solutions of type-IIB supergravity with general fluxes, hep-th/0403220.

[28] K. Pilch and N.P. Warner, Generalizing the $N=2$ supersymmetric RG flow solution of IIB supergravity, Nucl. Phys. B 675 (2003) 99 hep-th/0306098.

[29] K. Pilch and N.P. Warner, $N=1$ supersymmetric solutions of IIB supergravity from Killing spinors, hep-th/0403005.

[30] S. Fidanza, R. Minasian and A. Tomasiello, Mirror symmetric SU(3)-structure manifolds with NS fluxes, hep-th/0311122.

[31] J.H. Schwarz and P.C. West, Symmetries and transformations of chiral $N=2 D=10$ supergravity, Phys. Lett. B 126 (1983) 301.

[32] J.H. Schwarz, Covariant field equations of chiral $N=2 D=10$ supergravity, Nucl. Phys. B 226 (1983) 269.

[33] E. Bergshoeff, M. de Roo, B. Janssen and T. Ortin, The super D9-brane and its truncations, Nucl. Phys. B 550 (1999) 289 hep-th/9901055.

[34] S.F. Hassan, T-duality, space-time spinors and RR fields in curved backgrounds, Nucl. Phys. B 568 (2000) 145 hep-th/9907152.

[35] C.N. Gowdigere, D. Nemeschansky and N.P. Warner, Supersymmetric solutions with fluxes from algebraic Killing spinors, Adv. Theor. Math. Phys. 7 (2004) 787 [ep-th/0306097.

[36] D. Nemeschansky and N.P. Warner, A family of M-theory flows with four supersymmetries, hep-th/0403006.

[37] J.M. Maldacena and C. Núñez, Towards the large- $N$ limit of pure $N=1$ super Yang-Mills, Phys. Rev. Lett. 86 (2001) 588 hep-th/0008001. 
[38] I.R. Klebanov and M.J. Strassler, Supergravity and a confining gauge theory: duality cascades and $\chi S B$-resolution of naked singularities, J. High Energy Phys. 08 (2000) 052 hep-th/0007191.

[39] G. Papadopoulos and A.A. Tseytlin, Complex geometry of conifolds and 5-brane wrapped on 2-sphere, Class. and Quant. Grav. 18 (2001) 1333 hep-th/0012034.

[40] M. Cederwall, U. Gran, M. Nielsen and B.E.W. Nilsson, $(p, q)$ 5-branes in non-zero B-field, Ð. High Energy Phys. 01 (2000) 037 hep-th/9912106.

[41] I. Mitra and S. Roy, (NS5, Dp) and (NS5, D ( $p+2), D p)$ bound states of type-IIB and type-IIA string theories, J. High Energy Phys. 02 (2001) 026 hep-th/0011236.

[42] I. Mitra and S. Roy, (NS5, D5, D3) bound state, OD3, OD5 limits and $\mathrm{SL}(2, \mathbb{Z})$ duality, Phys. Rev. D 65 (2002) 106001 hep-th/0107127.

[43] U. Gran and M. Nielsen, On the equivalence of bound state solutions, Int. J. Mod. Phys. A 18 (2003) 3469 hep-th/0108113.

[44] P. Candelas and D.J. Raine, Spontaneous compactification and supersymmetry in D $=11$ supergravity, Nucl. Phys. B 248 (1984) 415. 